\title{
ORIGINAL ARTICLE \\ Prediction of outcome in newly diagnosed myeloma: a meta-analysis of the molecular profiles of 1905 trial patients
}

\author{
V Shah ${ }^{1}$, AL Sherborne ${ }^{1}$, BA Walker ${ }^{2}$, DC Johnson ${ }^{1}$, EM Boyle ${ }^{3}$, S Ellis ${ }^{1}$, DB Begum ${ }^{1}$, PZ Proszek ${ }^{1}$, JR Jones ${ }^{1}$, C Pawlyn ${ }^{1}$, S Savola ${ }^{4}$, \\ MW Jenner ${ }^{5}$, MT Drayson ${ }^{6}$, RG Owen ${ }^{7}$, RS Houlston ${ }^{1}$, DA Cairns ${ }^{8}$, WM Gregory ${ }^{8}$, G Cook ${ }^{9}$, FE Davies ${ }^{2}$, GH Jackson ${ }^{10}$, GJ Morgan $^{2}$ and \\ MF Kaiser ${ }^{1}$ on behalf of the NCRI Haemato-oncology CSG
}

Robust establishment of survival in multiple myeloma (MM) and its relationship to recurrent genetic aberrations is required as outcomes are variable despite apparent similar staging. We assayed copy number alterations (CNA) and translocations in 1036 patients from the NCRI Myeloma XI trial and linked these to overall survival (OS) and progression-free survival. Through a meta-anlysis of these data with data from MRC Myeloma IX trial, totalling 1905 newly diagnosed MM patients (NDMM), we confirm the association of $\mathrm{t}(4 ; 14), \mathrm{t}(14 ; 16), \mathrm{t}(14 ; 20)$, del(17p) and gain(1q21) with poor prognosis with hazard ratios (HRs) for OS of $1.60\left(P=4.77 \times 10^{-7}\right), 1.74(P=0.0005), 1.90(P=0.0089), 2.10\left(P=8.86 \times 10^{-14}\right)$ and $1.68\left(P=2.18 \times 10^{-14}\right)$, respectively. Patients with 'double-hit' defined by co-occurrence of at least two adverse lesions have an especially poor prognosis with HRs for OS of $2.67\left(P=8.13 \times 10^{-27}\right)$ for all patients and $3.19\left(P=1.23 \times 10^{-18}\right)$ for intensively treated patients. Using comprehensive CNA and translocation profiling in Myeloma XI we also demonstrate a strong association between $\mathrm{t}(4 ; 14)$ and $B I R C 2 / B I R C 3$ deletion $\left(P=8.7 \times 10^{-15}\right)$, including homozygous deletion. Finally, we define distinct sub-groups of hyperdiploid MM, with either gain $(1 \mathrm{q} 21)$ and CCND2 overexpression $(P<0.0001)$ or gain $(11 \mathrm{q} 25)$ and CCND1 overexpression $(P<0.0001)$. Profiling multiple genetic lesions can identify MM patients likely to relapse early allowing stratification of treatment.

Leukemia (2018) 32, 102-110; doi:10.1038/leu.2017.179

\section{INTRODUCTION}

While survival for multiple myeloma (MM) has improved over the last decade with the introduction of immunomodulatory drugs and proteasome inhibitors most MM patients will still relapse. ${ }^{1}$ Upfront identification of patients who are likely to relapse early offers the prospect of intervening pre-emptively to maintain remission. Furthermore, identifying tumor sub-groups with targetable molecular dependencies has the potential to inform on biologically driven therapy.

Myeloma cells are typified by recurrent chromosomal aberrations, a number of which have been variously associated with poor prognosis, notably $\mathrm{t}(4 ; 14), \mathrm{t}(14 ; 16), \mathrm{t}(14 ; 20)$, deletion $17 \mathrm{p}$ and gain of $1 \mathrm{q}^{2}$. We and others have recently reported that the cooccurrence of multiple genetic lesions may have greater significance for predicting patient outcome than any single abnormality. ${ }^{3,4}$ Since many of the molecular abnormalities in MM are only present at relatively low frequency, robustly establishing the impact of molecular sub-classes on prognosis is contingent on the analysis of large patient series that have been uniformly treated.

Here we report a meta-analysis of the relationship between genetic profile and prognosis in newly diagnosed MM (NDMM) using data from two UK multi-center phase III clinical trials, totalling 1905 patients. This dataset includes previously generated data on the MRC Myeloma IX trial and an expanded analysis of the NCRI Myeloma XI trial. In addition, we analysed molecular copy number profiling in 1036 Myeloma XI patients to identify subgroups with molecular addictions that could be therapeutically targetable.,

\section{MATERIALS AND METHODS}

Myeloma XI trial patients

1036 patients with NDMM enrolled in the UK NCRI Myeloma XI phase III trial were molecularly profiled. Trial characteristics are described in Supplementary Methods. At the time of analysis, the trial endpoints have not been published. Median follow-up was 36.0 months. The study was undertaken with written informed consent from patients and ethical approval was obtained from the Oxfordshire Research Ethics Committee (MREC 17/09/09, ISRCTN49407852).

\section{Myeloma IX trial patients}

Detailed characteristics and main outcomes of MRC Myeloma IX have been reported previously and summarised in Supplementary Methods. ${ }^{7}$ The study was undertaken with written informed consent from patients and ethical approval was obtained from the MRC Leukaemia Data Monitoring and Ethics committee (MREC 02/08/95, ISRCTN68454111). For the present analysis we included data from 869 of the 1960 NDMM patients with available clinical and comprehensive cytogenetic data. ${ }^{3}$ Median follow-up

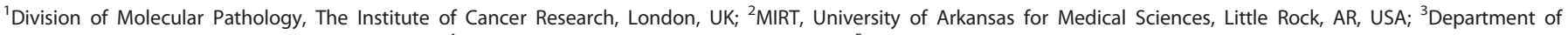

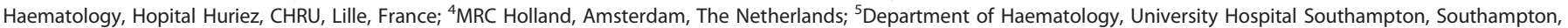

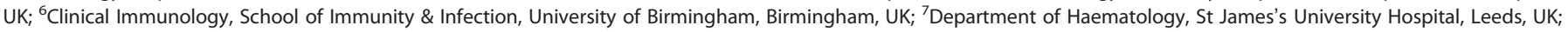

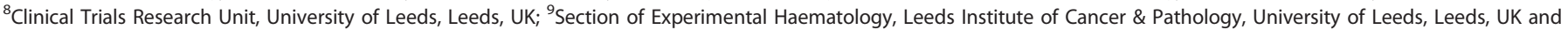

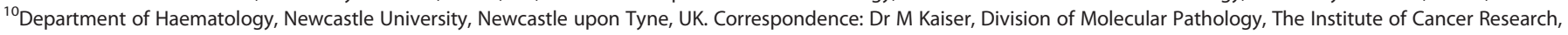
Sutton, London SM2 5NG, UK. 
for this group was 72 months. ${ }^{3,8}$ Accompanying gene expression and mapping array data have been previously published (GSE15695). ${ }^{6,9,10}$

\section{Samples}

For both trials myeloma cells from bone marrow aspirate samples were obtained at diagnosis and purified (>95\%) using immune-magnetic cell sorting (Miltenyi Biotec, Bergisch Gladbach, Germany). RNA and DNA were extracted using RNA/DNA mini kit or Allprep kits (QIAGEN) according to manufacturers' instructions.

\section{Copy number and translocation detection}

Technical details about fluorescence in situ profiling of Myeloma IX have been published previously. ${ }^{11}$ Myeloma XI cases were centrally analysed using MLPA and qRT-PCR. The SALSA MLPA P425-B1 MM probemix (MRC Holland, Amsterdam, The Netherlands) was used as previously described. ${ }^{12,13}$ The newly developed probemix X073-A1 was used to profile 1007 of the 1036 cases in an identical fashion (MLPA Probe Mix:
Supplementary Table 1). Copy number at each locus was determined as described previously. ${ }^{12,13}$

Multiplexed qRT-PCR was used to determine IGH translocation status using a translocation and cyclin D (TC)-classification based algorithm (Supplementary Methods), as previously described. ${ }^{10}$

\section{Statistical methods}

All statistical analyses were undertaken using $\mathrm{R}$ version 3.3 and the 'survival', 'rms', 'metafor', 'survC1', 'JAGS' and 'BayesMed' packages. ${ }^{14}$

Progression-free survival (PFS) was defined as the time from the date of randomization to progression, according to IMWG criteria, or death from any cause. Overall survival (OS) was defined as the time from the date of randomization to death from any cause. Kaplan-Meier survival curves were generated and the homogeneity between groups was evaluated with the log-rank test. Cox regression analysis was used to estimate hazard ratios (HRs) and respective 95\% confidence intervals (CI) and adjustment for variables was performed by multivariable analysis. Fixed effects metaanalysis was performed using individual patient data. Correlations between structural aberrations were analysed using Bayesian inference. A Bayes

Table 1. Clinical characteristics and frequency of genetic aberrations in myeloma IX and myeloma XI trial patients

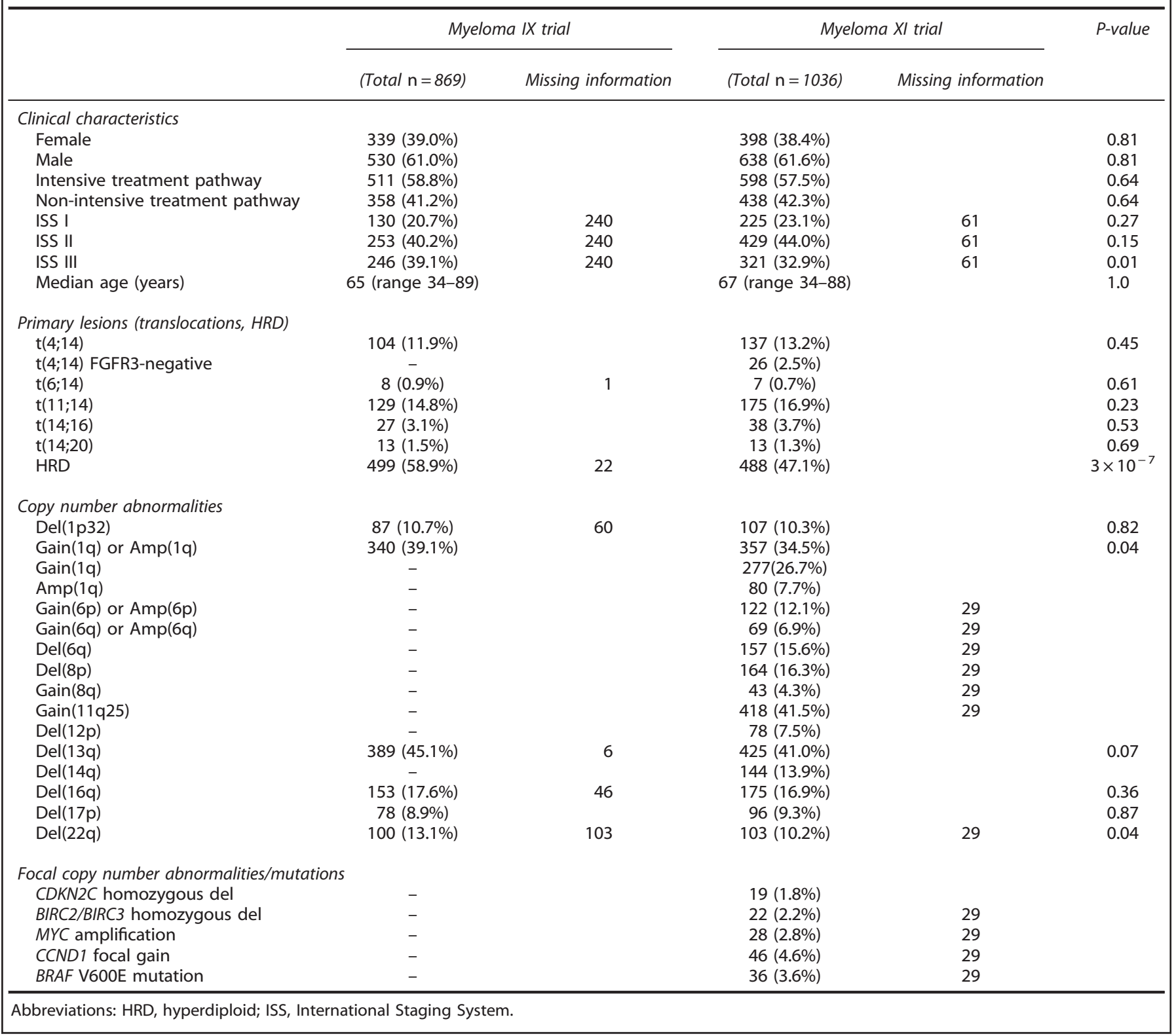


Table 2. Relationship between genetic abnormalities and patient survival

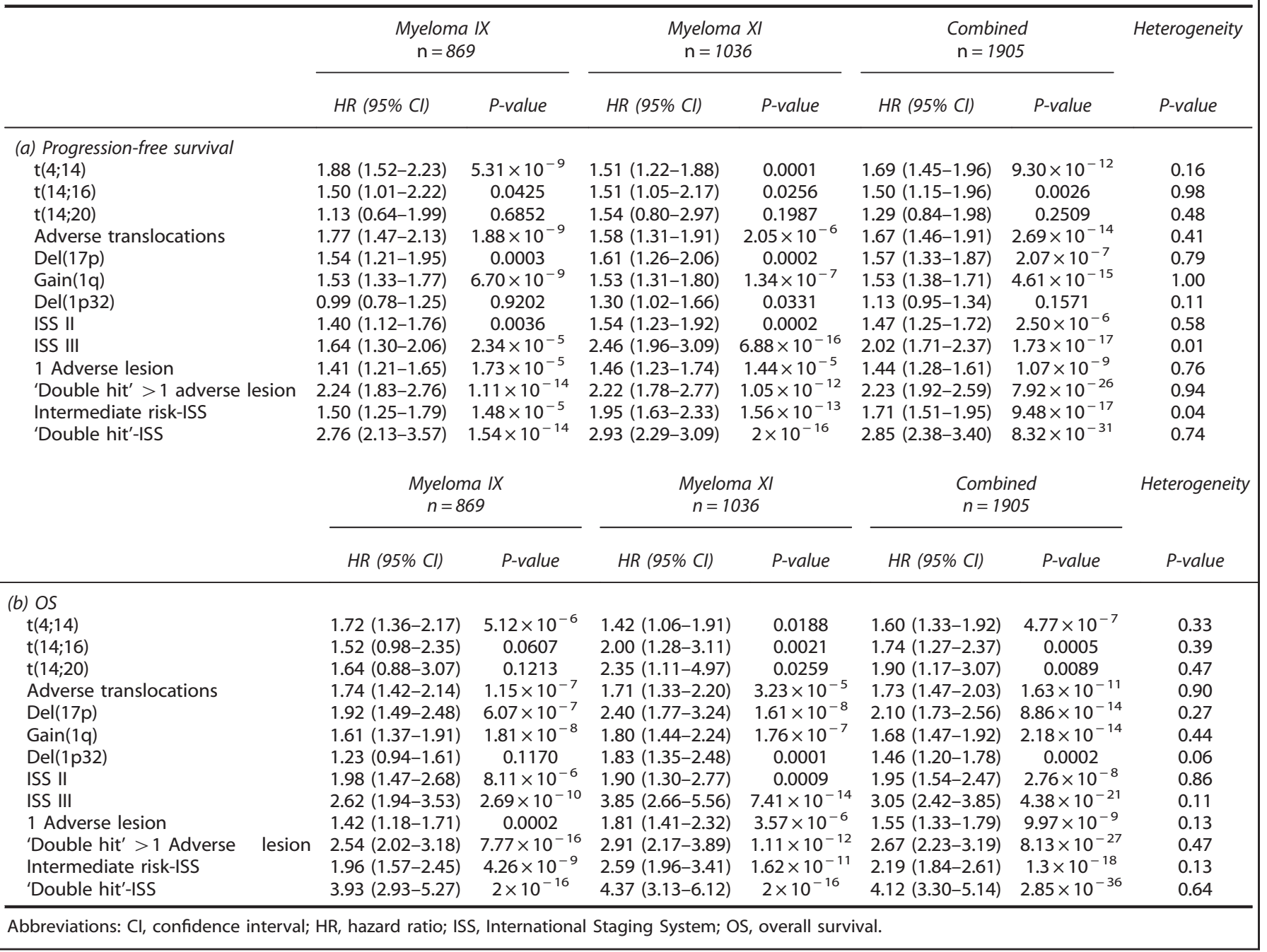

factor $\left(\mathrm{BF}_{01}\right)$ of $\mathrm{BF}_{01}<0.01$ was considered significant. The association between categorical variables was examined using the Fisher exact test. The association between myeloma subtype and gene expression was assessed using the Mann-Whitney test. A two-sided $P$-value $<0.05$ was considered significant.

\section{RESULTS}

Descriptive patient characteristics and structural aberrations The clinical characteristics of the 1036 newly profiled Myeloma XI trial patients and the 869 Myeloma Trial IX patients are detailed in Table 1. Overall there were no significant differences between trial patients in terms of gender, age and proportion that had been in receipt of intensive/non-intensive therapy. Although the frequencies of the primary IGH translocations, del(17p), del(1p32), del(13q) and $\operatorname{del}(16 q)$ in tumours were similar in Myeloma IX and XI trial patients, a higher proportion of Myeloma IX patients had hyperdiploidy (HRD), gain(1q) and del(22q) (Table 1). Amongst Myeloma XI trial patients, homozygous deletion of CDKN2C (1p32), BIRC2/BIRC3 (11q22) and amplification of CKS1B (1q21) and MYC (8q24) were the commonest focal homozygous copy number changes, which were seen at similar frequencies to those previously reported (Table 1$).^{15}$
Relationship between cytogenetic aberrations and survival

In both trial series, the archetypical high-risk lesions del(17p), gain (1q) and t(4;14) were each significantly associated with shorter PFS and OS (Table 2). In the combined analysis, respective HR for OS were 2.1 for $\operatorname{del}(17 p) \quad\left(P=8.86 \times 10^{-14}\right), 1.68$ for gain(1q) $\left(P=2.18 \times 10^{-14}\right)$ and 1.60 for $\mathrm{t}(4 ; 14) \quad\left(P=4.77 \times 10^{-7}\right.$; Table 2; Supplementary Figures 1 and 2$)$. In addition, the $t(14 ; 16)$ and $t$ $(14 ; 20)$ translocations involving $M A F$ and $M A F B$ were also associated with shorter OS with respective HRs of 1.74 $(P=0.0005)$ and HR $1.90 \quad(P=0.0089)$. Respective inference $C$-statistic estimates for adequacy of risk prediction are shown in Supplementary Tables 5 and 6.

Deletion of $1 \mathrm{p} 32$ (CDKN2C) was significantly associated with shorter OS (HR 1.46; $P=0.0002$; Table 2). This association was confined to patients in receipt of intensive treatment (in the combined analysis: HR $1.89 ; P=1.23 \times 10^{-5}$ vs HR $1.05 ; P=0.72$ for non-intensive treatment). The association of del(1p32) with OS was independent from gain(1q21) by multivariable analysis $(P<0.05)$ in the intensive treatment groups of both trials.

To examine the relationship between 1q21 status and outcome in more detail we sub-classified Myeloma XI patients $(n=1036)$ by diploid vs gain vs amplification status. 1q21 gain was confirmed as a high-risk lesion and was associated with significantly shorter PFS (HR 1.56; $P=3.53 \times 10^{-7}$ ) and OS (HR 1.67; $P=3.30 \times 10^{-5}$ ) than 
a

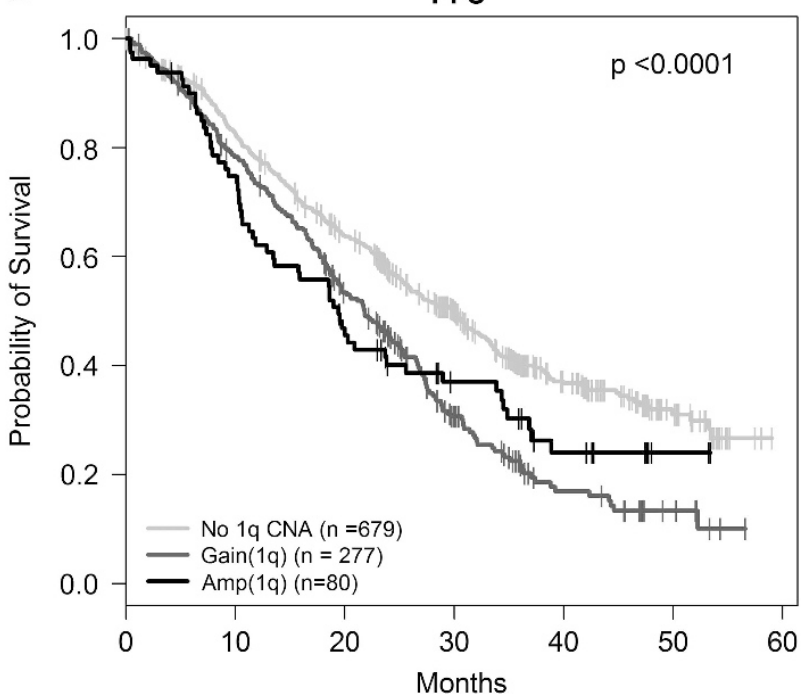

b

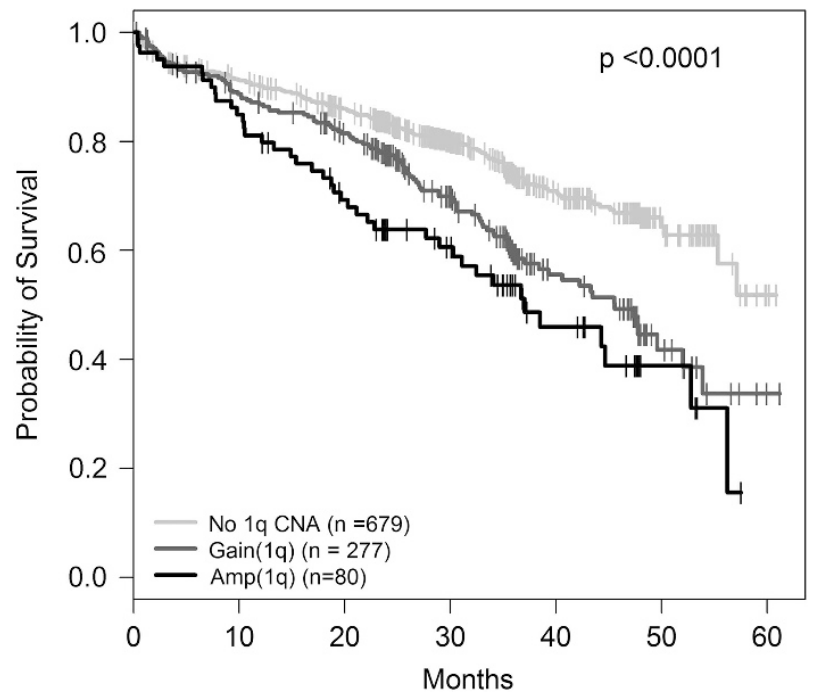

Figure 1. Chromosome 1q21 copy number status and outcome in Myeloma XI. Kaplan-Meier curves and log-rank $P$-values for (a) PFS (b) OS for normal vs gain vs amplification of $1 \mathrm{q} 21$.

normal 1q copy number status. Amp(1q) was also associated with shorter PFS (HR 1.44; $P=0.01$ ) and OS (HR 2.28; $P=2.32 \times 10^{-6}$ ) compared to normal $1 \mathrm{q}$, but there was no significant difference to gain(1q) (PFS: HR 0.91; $P=0.54$; OS: HR 1.36; $P=0.09$ for OS). Median PFS was 19.4 vs 21.8 vs 30.1 months $(P<0.0001)$ and 24 -months OS 63.8 vs 77.5 vs $83.5 \%(P<0.0001)$ for amp(1q), gain(1q) and normal 1q, respectively (Figure 1; Supplementary Table 7).

'Double-hit' as a high-risk classifier

We next examined the impact of a 'double-hit' based on the cooccurrence of at least any two of the following: (1) Adverse translocations $\mathrm{t}(4 ; 14), \mathrm{t}(14 ; 16), \mathrm{t}(14 ; 20)$; (2) gain $(1 \mathrm{q})$; (3) del(17p). ${ }^{3}$ For Myeloma XI the three risk groups, defined by 'double-hit', 1 or no adverse lesions, were associated with median PFS of 17.0, 24.2 and 31.1 months (log-rank $P=5.7 \times 10^{-13}$ ), with corresponding median 24-months OS of $66.1,76.6$ and $86.4 \%\left(P=4.4 \times 10^{-13}\right)$. These findings were consistent with Myeloma IX (Table 2). In the combined analysis of all 1905 patients the HR for 'double-hit' was 2.23 for PFS $\left(P=7.92 \times 10^{-26}\right)$ and 2.67 for OS $\left(P=8.13 \times 10^{-27}\right.$; Table 2; Supplementary Figures 1 and 2). Similarly to Myeloma IX, the 'triple-hit' of an adverse translocation, Gain(1q) and del(17p) was associated with a very short median OS of 19 months with a HR of $6.23\left(P=1.31 \times 10^{-7}\right)$ vs no adverse lesion (Supplementary Figure 5).

In both Myeloma IX and XI trials the impact of a 'double-hit' on patient outcome was independent of International Staging System (ISS; Supplementary Table 3). Moreover, integration of ISS and genetic risk defined 'double-hit'-ISS ultra high risk (ISS II or III and 'double-hit'; 12.0\%), intermediate risk (ISS I and 'double-hit'; ISS II and 1 adverse lesion; ISS III and no or 1 adverse lesion; 44.1\%) and favourable risk groups (ISS I and no or 1 adverse lesion; ISS II and no adverse lesion; 43.9\%). 'double hit'-ISS ultra high risk was associated with HR $2.85\left(P=8.32 \times 10^{-31}\right)$ for PFS and HR 4.12 $\left(P=2.85 \times 10^{-36}\right)$ for OS in the meta-analysis (Table 2$)$.

Genetic markers and survival in intensively treated patients Since young and fit patients are most likely to be considered for intensified combination therapy, we subsequently focused on the relationship between molecular profile and survival of this subgroup of Myeloma XI $(n=598)$ and Myeloma IX $(n=511)$ patients.

In these 1109 intensively treated patients, del(17p), gain(1q) and $\mathrm{t}(4 ; 14)$ were consistently associated with shorter PFS and OS; combined HRs of $2.65\left(3.04 \times 10^{-12}\right), 1.77\left(1.65 \times 10^{-8}\right)$ and 1.87 $\left(7.62 \times 10^{-7}\right)$, respectively (Table 3; Supplementary Figures 3 and 4). In this group, $t(14 ; 16)$ was associated with shorter PFS (HR 1.80; $P=0.0021)$ and OS (HR 1.82; $P=0.013)$. The $t(14 ; 20)$ was not associated with adverse PFS or OS, but the lesion was only present in eight Myeloma IX and five Myeloma XI cases (Table 3; Supplementary Figures 3 and 4).

In Myeloma XI, the groups with a 'double-hit', 1 adverse or no adverse lesion were associated with median PFS of 19.7, 30.9 and 44.8 months (log-rank $P=2.5 \times 10^{-13}$ ) and 24-months OS of 72.3, 86.2 and $92.2 \%\left(P=1.6 \times 10^{-10}\right.$; Supplementary Figure 3$)$. By metaanalysis, intensively treated patients with a 'double-hit' had a HR for PFS of $2.61 \quad\left(P=1.07 \times 10^{-20}\right)$ and HR for OS of 3.19 $\left(P=1.23 \times 10^{-18}\right.$; Table 3$)$. Survival time increased for all risk groups of intensively treated patients in Myeloma XI compared to Myeloma IX (median PFS: 14.4, 21.9 and 30.8 months; 24 month OS: 63.9, 75.4 and $86.0 \%$, respectively). Median PFS was 5.3 months longer for 'double-hit' in Myeloma XI vs IX, but 14 months longer for the group without any risk lesion.

On the basis of clinical and genetic information (Supplementary Table 4) the 'double-hit'-ISS ultra high-risk group comprising $12.5 \%$ of patients were associated with a HR of 3.11 $\left(P=1.59 \times 10^{-20}\right)$ for PFS and HR $4.79\left(P=5.10 \times 10^{-23}\right)$ for OS.

Associations of copy number changes with translocations and targetable lesions

We next focused on genetic sub-groups of MM that could be specifically targetable using copy number and translocation data on the 1036 Myeloma XI patients. Figure 2 provides an overview of correlations between CNA and translocations (Supplementary Table 2). Of particular note was a relationship between NFKBpathway CNA and translocation groups.

Potentially targetable NFkB-pathway gene deletions are common in myeloma

Deletions of NFKB-pathway modulating genes TNFAIP3, BIRC2/ BIRC3, TRAF3 or CYLD were identified in $16.6,4.8,13.9$ and $16.9 \%$ of Myeloma XI cases, respectively. Nearly half of all tumors (43.2\%) harbored an NFKB-pathway gene abnormality. Overall, a deletion of more than one NFKB-pathway gene was detectable in $9.7 \%$ of 
Table 3. Relationship between genetic abnormalities and patient survival for intensively treated patients

\begin{tabular}{|c|c|c|c|c|c|c|c|}
\hline & \multicolumn{2}{|c|}{$\begin{array}{c}\text { Myeloma IX } \\
n=511\end{array}$} & \multicolumn{2}{|c|}{$\begin{array}{c}\text { Myeloma XI } \\
n=598\end{array}$} & \multicolumn{2}{|c|}{$\begin{array}{c}\text { Combined } \\
n=1109\end{array}$} & \multirow{2}{*}{$\begin{array}{c}\text { Heterogeneity } \\
\text { P-value }\end{array}$} \\
\hline & $H R(95 \% \mathrm{Cl})$ & $P$-value & $H R(95 \%$ Cl) & $P$-value & $H R(95 \%$ Cl) & P-value & \\
\hline \multicolumn{8}{|l|}{ (a) Progression-free survival } \\
\hline$t(4 ; 14)$ & $1.96(1.49-2.59)$ & $1.80 \times 10^{-6}$ & $2.03(1.56-2.64)$ & $2.18 \times 10^{-7}$ & $2.00(1.65-2.42)$ & $1.85 \times 10^{-12}$ & 0.88 \\
\hline$t(14 ; 16)$ & $1.60(0.96-2.69)$ & 0.0729 & $2.03(1.19-3.47)$ & 0.0099 & $1.80(1.23-2.60)$ & 0.0021 & 0.54 \\
\hline$t(14 ; 20)$ & $0.96(0.46-2.03)$ & 0.9192 & $0.64(0.09-4.54)$ & 0.6524 & $0.91(0.45-1.84)$ & 0.7987 & 0.70 \\
\hline Gain(1q) & $1.48(1.22-1.80)$ & $7.44 \times 10^{-5}$ & $1.65(1.31-2.07)$ & $2.03 \times 10^{-5}$ & $1.55(1.34-1.80)$ & $7.59 \times 10^{-9}$ & 0.49 \\
\hline $\operatorname{Del}(1 \mathrm{p} 32)$ & $1.05(0.76-1.47)$ & 0.7556 & $1.48(1.04-2.09)$ & 0.0286 & $1.23(0.97-1.57)$ & 0.0833 & 0.17 \\
\hline ISS II & $1.34(1.01-1.77)$ & 0.0409 & $1.48(1.11-1.99)$ & 0.0085 & $1.40(1.15-1.72)$ & 0.0009 & 0.61 \\
\hline ISS III & $1.43(1.07-1.91)$ & 0.0168 & $2.20(1.61-3.01)$ & $7.88 \times 10^{-7}$ & $1.74(1.40-2.16)$ & $3.11 \times 10^{-7}$ & 0.04 \\
\hline 1 Adverse lesion & $1.50(1.21-1.85)$ & 0.0002 & $1.49(1.15-1.93)$ & 0.0024 & $1.50(1.27-1.76)$ & $1.36 \times 10^{-6}$ & 0.99 \\
\hline 'Double it' $^{\prime}>1$ adverse lesion & $2.31(1.75-3.05)$ & $3.67 \times 10^{-14}$ & $3.00(2.24-4.02)$ & $2.17 \times 10^{-13}$ & $2.61(2.13-3.20)$ & $1.07 \times 10^{-20}$ & 0.21 \\
\hline Intermediate risk-ISS & $1.47(1.16-1.86)$ & 0.0015 & $1.87(1.45-2.41)$ & $1.45 \times 10^{-6}$ & $1.64(1.38-1.95)$ & $2.10 \times 10^{-8}$ & 0.17 \\
\hline 'Double hit'-ISS & $H R(95 \% \mathrm{Cl})$ & $P$-value & $H R(95 \%$ Cl) & $P$-value & $H R(95 \% \mathrm{Cl})$ & $P$-value & $P$-value \\
\hline \multicolumn{8}{|l|}{ (b) OS } \\
\hline$t(4 ; 14)$ & $1.74(1.26-2.41)$ & 0.0008 & $2.09(1.41-3.07)$ & 0.0002 & $1.87(1.46-2.40)$ & $7.62 \times 10^{-7}$ & 0.49 \\
\hline$t(14 ; 16)$ & $1.51(0.79-2.84)$ & 0.2059 & $2.31(1.13-4.73)$ & 0.0218 & $1.82(1.13-2.92)$ & 0.01359 & 0.38 \\
\hline $\mathrm{t}(14 ; 20)$ & $1.44(0.59-3.49)$ & 0.4181 & $1.91(0.26-13.70)$ & 0.5219 & $1.51(0.67-3.39)$ & 0.3169 & 0.80 \\
\hline Adverse translocations & $1.74(1.30-2.33)$ & 0.0002 & $2.30(1.60-3.31)$ & $6.99 \times 10^{-6}$ & $1.94(1.55-2.43)$ & $9.91 \times 10^{-9}$ & 0.24 \\
\hline $\operatorname{Del}(17 p)$ & $2.31(1.61-3.31)$ & $5.87 \times 10^{-6}$ & $3.19(2.10-4.85)$ & $5.74 \times 10^{-8}$ & $2.65(2.01-3.48)$ & $3.04 \times 10^{-12}$ & 0.25 \\
\hline Gain(1q) & $1.79(1.40-2.27)$ & $2.33 \times 10^{-6}$ & $1.72(1.22-2.43)$ & 0.0020 & $1.77(1.45-2.15)$ & $1.65 \times 10^{-8}$ & 0.86 \\
\hline $\operatorname{Del}(1 p 32)$ & $1.84(1.28-2.64)$ & 0.0010 & $1.99(1.25-3.18)$ & 0.0037 & $1.89(1.42-2.52)$ & $1.23 \times 10^{-5}$ & 0.78 \\
\hline ISS II & $1.96(1.32-2.90)$ & 0.0008 & $1.88(1.14-3.11)$ & 0.0140 & $1.92(1.41-2.63)$ & $3.27 \times 10^{-5}$ & 0.90 \\
\hline ISS III & $2.56(1.72-3.81)$ & $3.59 \times 10^{-6}$ & $3.22(1.94-5.35)$ & $6.56 \times 10^{-6}$ & $2.79(2.04-3.81)$ & $1.30 \times 10^{-10}$ & 0.49 \\
\hline
\end{tabular}

tumors and in $42.4 \%$ of these cases involved deletions of both TNFAIP3 and CYLD.

$\mathrm{t}(4 ; 14)$ myeloma is associated with BIRC2/BIRC3 deletions We identified BIRC NFKB-pathway deletions to be enriched in t(4;14) MM (Figure 2). Specifically, 29/135 (21.5\%) t(4;14) vs 20/852 (2.3\%) non-t $(4 ; 14) \quad\left(P=8.7 \times 10^{-15}\right)$ tumors were BIRC2/BIRC3 deleted. Intriguingly, homozygous $B I R C 2 / B I R C 3$ deletions were present in $15 / 135(11 \%)$ of $t(4 ; 14)$ as compared to $7 / 872$ non-t $(4 ; 14)$ tumors $\left(0.8 \% ; \quad \mathrm{BF}_{01}=4.3 \times 10^{-12} ; \quad P=1.0 \times 10^{-8}\right.$; Supplementary Table 2). Almost all $t(4 ; 14)$ tumors $(28 / 29)$ with any $B I R C 2 / B I R C 3$ deletion expressed FGFR3, more than expected $(P=0.015)$. Deletions of TRAF3 (14q32) seen in $t(4 ; 14)$ were mutually exclusive of $B I R C$ deletions $(P=0.016)$ and more than expected FGFR3-negative: 20/29 (69\%; $P=0.0001$ ). In contrast, deletions of CYLD were generally significantly under-represented in the $\mathrm{t}(4 ; 14)$ group $(6 / 137 ; 4.4 \%)$ as compared with non-t $(4 ; 14)$ $\left(169 / 899 ; 18.8 \% ; \mathrm{BF}=0.007 ; P=4.0 \times 10^{-6}\right)$.

High-risk and hypodiploidy-associated lesions

The $t(4 ; 14)$ subgroup was significantly associated with hypodiploidy lesions (HYL) del(12p) $\left(B F=1.1 \times 10^{-4}\right)$, $\quad$ del $(13 q)$ $\left(\mathrm{BF}=1.1 \times 10^{-25}\right)$ and $\operatorname{del}(22 \mathrm{q})\left(\mathrm{BF}=1.1 \times 10^{-7}\right.$; Figure 2$){ }^{16}$ Deletion of $17 p$ was also associated with deletions of $12 p(B F=0.0004)$,
$13 q\left(B F=3.7 \times 10^{-6}\right)$ and $22 q(B F=0.0076)$, but there was no correlation between del(17p) and $t(4 ; 14)$. MM with $t(4 ; 14)$ was associated with gain $(1 \mathrm{q})\left(B F=3.0 \times 10^{-8}\right)$, but not with del $(1 p 32)$, which was only significantly correlated with del $(8 q)(B F=0.0009)$ and del $(16 q 23)(B F=0.0003)$. In contrast, $t(11 ; 14)$ and HRD cases, the latter defined by extra copies of any two of chromosomes 5, 9 or 15 , were negatively associated with gain $(1 \mathrm{q})\left(\mathrm{BF}=1.6 \times 10^{-3}\right.$ and $\mathrm{BF}=0.06$, respectively). Collectively, HRD cases were negatively associated with $\operatorname{del}(13 q)\left(B F=1.9 \times 10^{-21}\right)$ and $\operatorname{del}(22 q)$ $(\mathrm{BF}=0.0002)$.

Molecular sub-classification of hyperdiploid myeloma We noted heterogeneity within the HRD subgroup in terms of cooccurrence of lesions. Although HRD as a whole group was strongly correlated with gain(11q25) $\left(\mathrm{BF}=1.2 \times 10^{-66}\right)$, a subgroup lacking gain(11q25) was characterised by gain(1q) (Figure 3; Supplementary Figure 6).

Of the $488 \mathrm{HRD}$ cases in Myeloma XI, 68\% had gain(11q25) and $29 \%$ gain(1q). Both lesions co-occurred in $15 \%$ of HRD cases, less than expected $(B F=0.0004)$. Accordingly, most HRD patients could be classified as having gain(1q)-HRD, gain(11q25)-HRD or gain(1q) +gain(11q25)-HRD (Figure 2a). Gain(1q)-HRD was associated with overexpression of CCND2 and silenced CCND1 $(P<0.0001)$. In contrast, gain(11q25) was associated with CCND1 expression and 


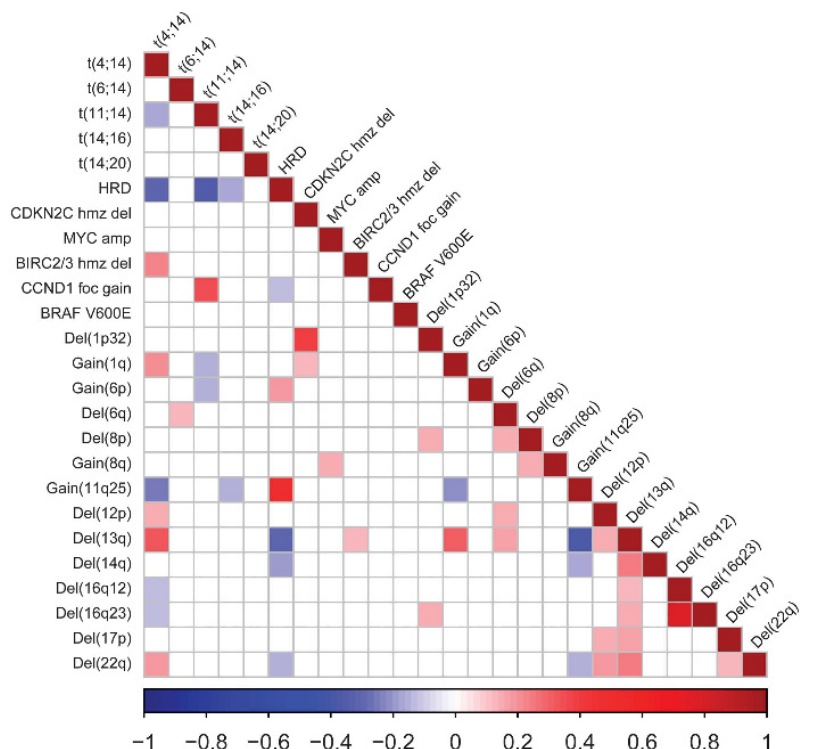

Figure 2. Associations between copy number aberrations and translocations in Myeloma XI. A Bayesian approach was used to identify all potential associations between genetic lesions. Significant interactions $(\mathrm{BF}<0.01)$ are colour-coded, red representing positive and blue negative associations. Correlation factors and Bayes Factors are provided in Supplementary Table 2. amp, amplification; foc gain, focal gain; hmz del, homozygous deletion.

silenced CCND2 $(P<0.0001)$. We validated this correlation between gain(1q)-HRD and CCND2 and gain(11q25)-HRD and CCND1 expression in the Myeloma IX dataset (Supplementary Figure 7). The TC classification-defined D1 and D2 sub-groups of HRD MM on the basis of CCND1 and CCND2 overexpression ${ }^{17}$ and our findings suggest similarity between gain(11q25)-HRD and the D1, gain(1q)-HRD and the D2 and gain(1q)+gain(11q)-HRD and the D1+D2 TC classification subgroup.

Further differences between the HRD subtypes were noted: $13 q$ was deleted in $41.1 \%(58 / 141)$ of gain(1q)-HRD $\left(B F=6.0 \times 10^{-6}\right.$; $P<0.0001)$, but only in $15.4 \%(50 / 325)$ of gain(11q25)-HRD $\left(\mathrm{BF}=5.5 \times 10^{-11} ; P<0.0001\right)$. We validated this finding in the Myeloma IX dataset, where del(13q) was also positively associated with gain(1q)-HRD ( $P=0.024)$ and negatively associated with gain (11q25) $(P=0.041)$.

\section{Prognostic impact of molecular sub-groups in HRD}

Gain of 1q, del(1p32) and del(17p) was associated with shorter OS (HR 1.81, $P=0.001 ; \operatorname{HR} 2.44, P=0.0004 ; \mathrm{HR} 1.89, P=0.022$; respectively) in the $488 \mathrm{HRD}$ cases. Gain(1q) and del(1p32) but not del(17p) was also associated with shorter PFS (HR 1.56, $P=0.0003 ;$ HR 1.66, $P=0.005 ;$ HR $1.30, P=0.23$ respectively; (Supplementary Table 8, Supplementary Figure 8)). Gain of $11 q 25$, del(13q) and del(22q) were not associated with shorter OS or PFS. At least one of the lesions gain(1q), del(1p32) or del (17p) were present in $39.3 \%$ (192/488) of HRD cases, defining a risk population with significantly shorter PFS $\left(P=4.9 \times 10^{-6}\right)$ and OS $\left(P=2.7 \times 10^{-6}\right.$; Supplementary Figure 9$)$ compared to HRD MM lacking any of these lesions. Interestingly, the $28.5 \%$ of all patients (296/1036) that had HRD without any demonstrable adverse lesion, had the longest survival of all sub-groups, indeed longer than those with $\mathrm{t}(11 ; 14) \mathrm{MM}$ (Figure 4).

\section{DISCUSSION}

Our analysis confirms the association with outcome in MM for the archetypical high-risk lesions del(17p), gain(1q) and adverse translocations and emphasises the importance of 'double-hit' as a risk biomarker. Importantly, we demonstrate that this information can be combined with the ISS to further refine risk prediction.,18-21 To our knowledge, this study represents the largest analysis investigating the additive effect of multiple genetic lesions on outcome in NDMM. Importantly, our analysis has been based on trials that recruited between 2003 and 2016, a timeframe during which treatment for MM has undergone significant change. 22 The consistent adverse impact of high-risk genetics on survival in Myeloma IX and XI is striking and highlights the need for intensified efforts to target the biology of high-risk disease. Although survival time increased for all risk groups in Myeloma XI vs IX, absolute improvement was smallest for the 'double-hit' high-risk group. Median PFS for 'double-hit' in Myeloma XI patients receiving intensive treatment was 19.7 months, meaning that about half of patients relapsed 12 months following autologous transplant.

Comprehensive assessment of the inter-relationship of CNAs and translocations in the Myeloma XI trial led to characterisation of genetic sub-groups with putative therapeutic relevance. We found that half of Myeloma XI tumors carried a deletion of NFKBpathway genes, and $10 \%$ of tumors had two co-occuring deletions. $^{23-25}$ Intriguingly, our data suggests NFKB-inducing kinase (NIK)-specific addiction of the $\mathrm{t}(4 ; 14)$ group: BIRC2/BIRC3 deletions, including homozygous deletions, were enriched in $\mathrm{t}(4 ; 14)$ tumours. The $\mathrm{t}(4 ; 14) \mathrm{MM}$ without $B I R C 2 / B I R C 3$ deletions were frequently TRAF3 deleted. BIRC2, BIRC3 and TRAF3 proteins all interact directly with NIK, suppressing NFKB-pathway activity. (25) $\mathrm{MM}$ cell lines with deletions of BIRC2/BIRC3 or TRAF3, predominantly $\mathrm{t}(4 ; 14)$, have high NIK levels and activated NFKBpathway signalling, as demonstrated by Keats et al. Recently, specific NIK inhibitors have been developed which might be used to target high-risk $\mathrm{t}(4 ; 14) \mathrm{MM}^{26-28}$ Virtually all BIRC2/3 deletions were found in FGFR3-positive tumors. They were mutually exclusive of TRAF3 deletions, which were present in FGFR3negative tumors, a pattern which may indicate convergent evolution. Deletions of FGFR3, which often occur as loss of der14 that includes TRAF3, may constitute 'collateral damage' of NIK addiction in $\mathrm{t}(4 ; 14) .15,24,25,29,30$

Although $\mathrm{t}(4 ; 14)$ and del(17p) were not correlated with each other, both groups were strongly associated with hypodiploidyassociated lesions del(12p), del(13q) and del(22q). ${ }^{16,17,31,32}$ This suggests the consequences of $t(4 ; 14)$ and del(17p) may share molecular mechanisms. Gain of chromosome 1q21 was strongly associated with $\mathrm{t}(4 ; 14)$, but not with del(17p). Gain(1q21) was confirmed as a high-risk lesion that is independent of del (1p32). ${ }^{33-35}$

HRD MM constitutes the largest genetic sub-group of patients, with substantial heterogeneity. ${ }^{17}$ We describe two sub-groups of HRD with either gain(11q25) and CCND1 biology or gain(1q21) and CCND2 overexpression. These groups are similar to the D1 and D2 sub-groups of the TC classification, which pioneered biologic classification of HRD MM. Application of the TC classification in routine diagnostics has unfortunately been restricted due to access limitations to array-based gene expression profiling. ${ }^{17}$ Pragmatic classification of HRD based on gain(11q25) and gain(1q) may facilitate sub-grouping in clinical practice and open opportunities for improving therapy for these patients. Recently, activity of bcl-2 inhibitors has been reported in CCND1-driven $\mathrm{t}(11 ; 14) \mathrm{MM}$, and CCND1-driven gain(11q25)-HRD may constitute another target group. ${ }^{36}$ We also found a high frequency of del (13q) in gain(1q)-HRD, in contrast to gain(11q25)-HRD. Interestingly, del(13q) and gain(1q) also frequently co-occur in $t(4 ; 14)$, suggesting similarities in the genetic sequelae of these pathogenetic groups. ${ }^{37}$ An inter-relationship between $\operatorname{del}(13 q)$ and gain (1q)-HRD was suggested based on GEP in the TC classification by Bergsagel et al., but has been demonstrated here for the first time on a DNA level. ${ }^{17,38}$ Moreover, HRD MM without any of risk lesions 
a

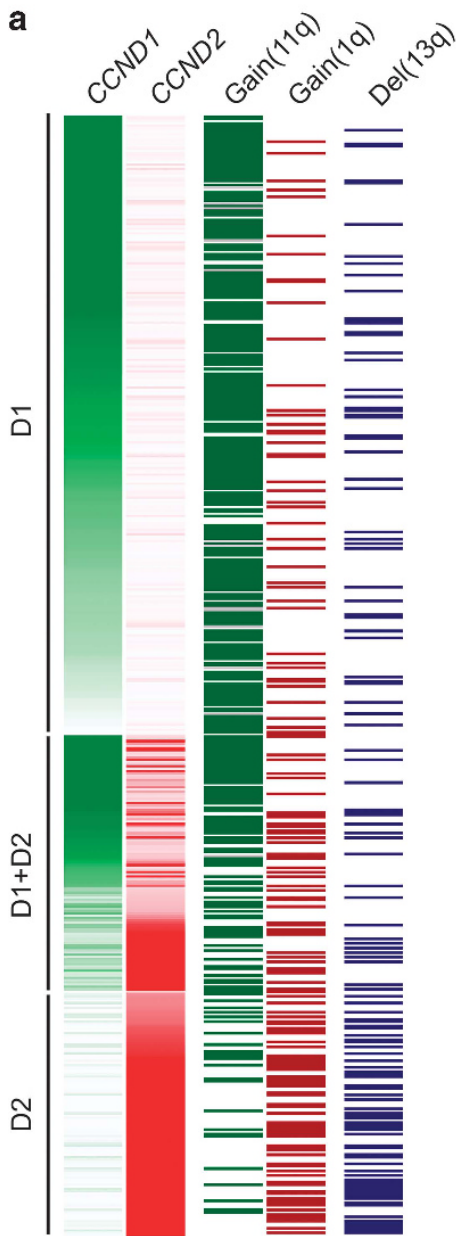

b

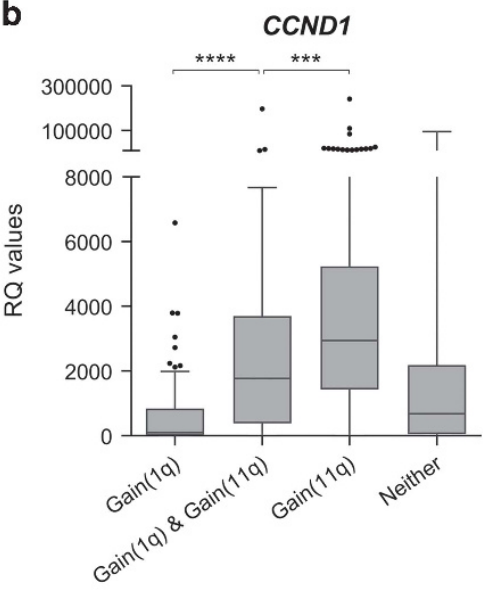

C

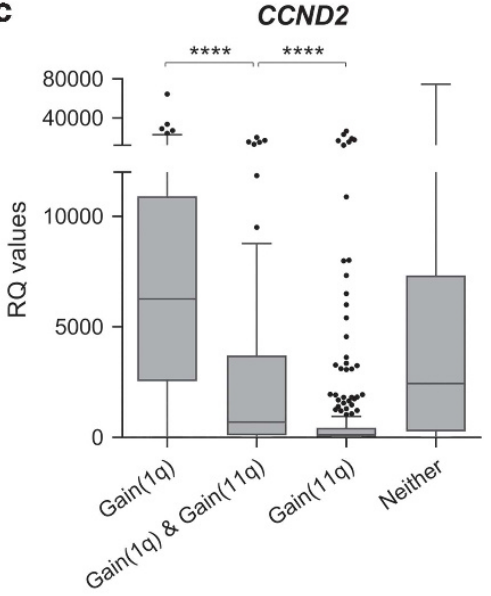

Figure 3. HRD genetic sub-groups in Myeloma XI. (a) Each row represents one of in total 1007 cases. Expression intensity is coded in green for CCND1 and red for CCND2 expression. Gain of $11 \mathrm{q} 25$ is shown in dark green, gain of $1 \mathrm{q}$ in dark red and deletion $13 \mathrm{q}$ in dark blue; white $=$ no abnormality detected. B+C. CCND1 (b) and CCND2 (c) qRT-PCR expression levels (relative quantitative RQ values, GAPDH normalised) for HRD cases with gain(1q), gain(1q)+gain(11q25), gain(11q25) or neither. Gene expression levels were significantly different for all possible groupwise comparisons (two-sided Mann-Whitney $U$ test; ${ }^{* * * *} P<0.0001 ;{ }^{* *} P<0.001$ ).

a

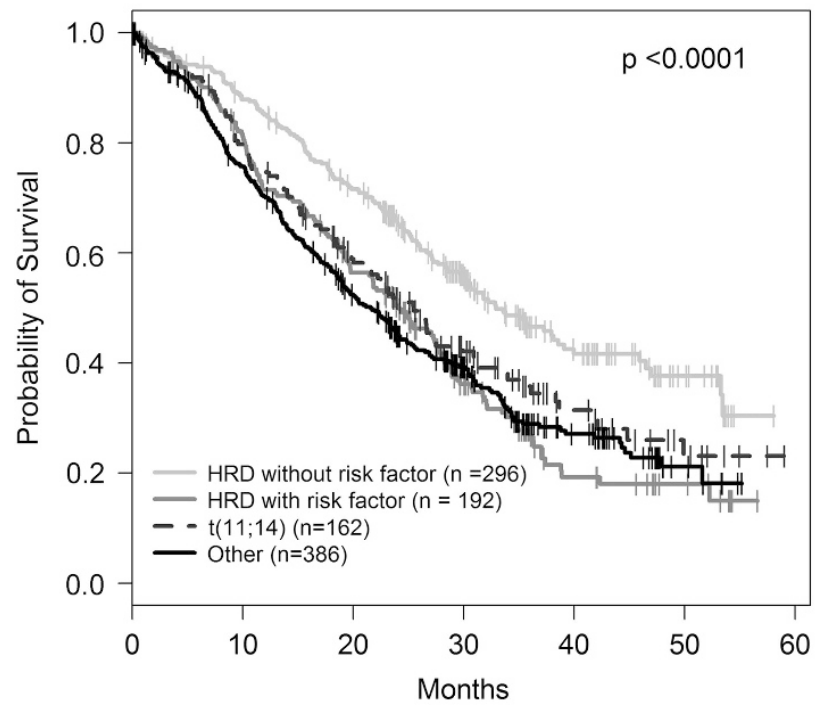

b

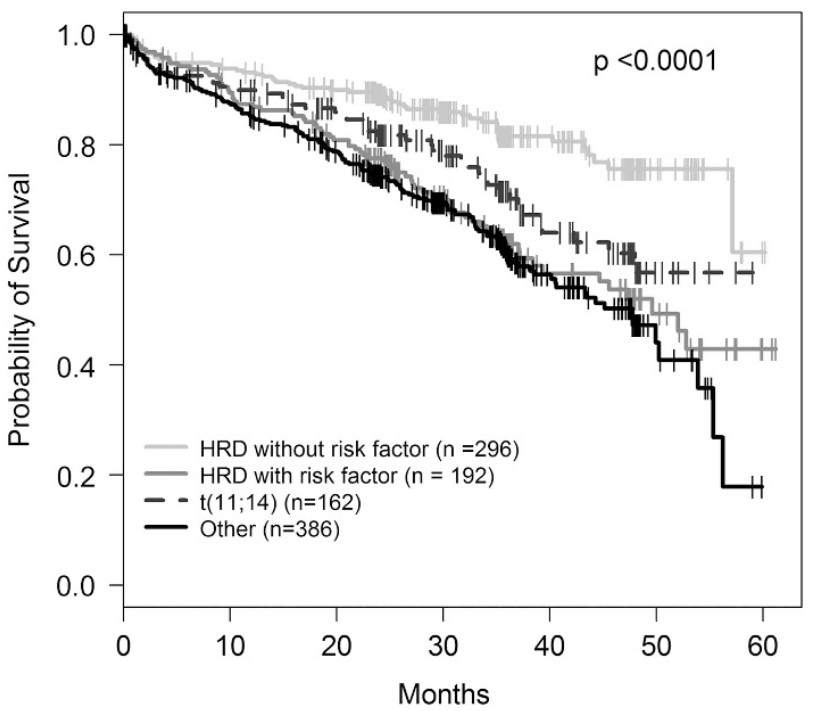

Figure 4. Survival in HRD MM with and without risk factors in Myeloma XI. Kaplan-Meier curves and log-rank $P$-values for (a) PFS (b) OS. 
gain(1q)-HRD, del(17p) and del(1p32) had longer remissions and survival than any other sub-group and may be sufficiently treated with single-novel agent/immunomodulatory drug-based approaches, potentially reducing additional side effects and costs of novel agent combinations. ${ }^{39-42}$

In summary, we demonstrate the utility of profiling multiple molecular genetic lesions to identify patients most likely to benefit from molecularly targeted therapies. The molecular tools used for profiling Myeloma XI are readily applicable within diagnostic settings and should therefore help implementing stratified treatment approaches as part of routine patient care.

\section{CONFLICT OF INTEREST}

Honoraria from Novartis, Pfizer, Takeda, Janssen by EMB; research support from Celgene by JRJ; consultancy and honoraria from Celgene, Novartis, Takeda by CP; SS was employed by MRC Holland; Honoraria and consultancy from Janssen, Novartis, Amgen, Takeda, Celgene by MWJ; Research funding: Janssen, Celgene; employment, equity ownership, royalties from Abingdon Health/Serascience by MTD; honoraria, consultancy from Celgene, Takeda, Janssen by RGO; Research funding from Celgene by DAC; Research Funding from Celgene by WMG; consultancy and research funding from Janssen, Celgene, Takeda Oncology, Sanofi, Amgen and BMS by GC; honoraria from Takeda-Millenium, Onyx/Amgen, Celgene and Janssen by FED; GHJ: honoraria and consultancy from Celgene, Takeda, MSD, Janssen, Roche andAmgen; research funding and travel support from Celgene and Takeda; honoraria, consultancy, research funding from Celgene, Takeda-Millennium, BMS by GJM; honoraria from Amgen, Celgene, Janssen: Consultancy for Takeda, Amgen, Chugai Pharma, BMS Janssen; Research Funding from Celgene by MFK. The remaining authors declare no conflict of interest.

\section{ACKNOWLEDGEMENTS}

We are grateful to the NCRI Haemato-oncology subgroup and to all investigators for recruiting patients to Myeloma XI. The principal investigators at the four top recruiting centres were Jindriska Lindsay (Kent and Canterbury Hospital), Nigel Russell (Nottingham University Hospital), Claire Chapman (Leicester Royal Infirmary) and Julie Blundell (Royal Cornwall Hospital). We thank the Clinical Trials Research Unit at The University of Leeds, Anna Waterhouse, Corrine Collett and Alina Striha for their diligence. We acknowledge the work of Bektas Aygun (MRC Holland) in the development and optimisation of the novel X073 MLPA assay. Finally, we are grateful to all patients whose participation made this study possible. This work was supported by Myeloma UK, Cancer Research UK CTAAC sample collection grants (C2470/A12136 and C2470/A17761) and a Cancer Research UK Biomarkers and Imaging Discovery and Development grant (C2470/A14261). We also acknowledge support from the National Institute of Health Biomedical Research Centre at the Royal Marsden Hospital.

\section{REFERENCES}

1 Kumar SK, Dispenzieri A, Lacy MQ, Gertz MA, Buadi FK, Pandey S et al. Continued improvement in survival in multiple myeloma: changes in early mortality and outcomes in older patients. Leukemia 2014; 28: 1122-1128.

2 Chng WJ, Dispenzieri A, Chim CS, Fonseca R, Goldschmidt H, Lentzsch S et al. IMWG consensus on risk stratification in multiple myeloma. Leukemia 2013; 28: 269-277.

3 Boyd KD, Ross FM, Chiecchio L, Dagrada GP, Konn ZJ, Tapper WJ et al. A novel prognostic model in myeloma based on co-segregating adverse FISH lesions and the ISS: analysis of patients treated in the MRC Myeloma IX trial. Leukemia 2012; 26: 349-355.

4 Avet-Loiseau H, Li C, Magrangeas F, Gouraud W, Charbonnel C, Harousseau JL et al. Prognostic significance of copy-number alterations in multiple myeloma. J Clin Oncol 2009; 27: 4585-4590.

5 Walker BA, Wardell CP, Murison A, Boyle EM, Begum DB, Dahir NM et al. APOBEC family mutational signatures are associated with poor prognosis translocations in multiple myeloma. Nat Commun 2015; 6: 6997.

6 Walker BA, Boyle EM, Wardell CP, Murison A, Begum DB, Dahir NM et al. Mutational spectrum, copy number changes, and outcome: results of a sequencing study of patients with newly diagnosed myeloma. J Clin Oncol 2015; 33: 3911-3920.

7 Morgan GJ, Child JA, Gregory WM, Szubert AJ, Cocks K, Bell SE et al. Effects of zoledronic acid versus clodronic acid on skeletal morbidity in patients with newly diagnosed multiple myeloma (MRC Myeloma IX): secondary outcomes from a randomised controlled trial. Lancet Oncol 2011; 12: 743-752.
8 Morgan GJ, Davies FE, Gregory WM, Bell SE, Szubert AJ, Cook G et al. Long-term follow-up of MRC Myeloma IX trial: survival outcomes with bisphosphonate and thalidomide treatment. Clin Cancer Res 2013; 19: 6030-6038.

9 Dickens NJ, Walker BA, Leone PE, Johnson DC, Brito JL, Zeisig A et al. Homozygous deletion mapping in myeloma samples identifies genes and an expression signature relevant to pathogenesis and outcome. Clin Cancer Res 2010; 16: 1856-1864.

10 Kaiser MF, Walker BA, Hockley SL, Begum DB, Wardell CP, Gonzalez D et al. A TC classification-based predictor for multiple myeloma using multiplexed real-time quantitative PCR. Leukemia 2013; 27: 1754-1757.

11 Ross FM, Chiecchio L, Dagrada G, Protheroe RK, Stockley DM, Harrison CJ et al. The $t(14 ; 20)$ is a poor prognostic factor in myeloma but is associated with long-term stable disease in monoclonal gammopathies of undetermined significance. Haematologica 2010; 95: 1221-1225.

12 Schwab CJ, Jones LR, Morrison H, Ryan SL, Yigittop H, Schouten JP et al. Evaluation of multiplex ligation-dependent probe amplification as a method for the detection of copy number abnormalities in B-cell precursor acute lymphoblastic leukemia. Genes Chromosomes Cancer 2010; 49: 1104-1113.

13 Boyle EM, Proszek PZ, Kaiser MF, Begum D, Dahir N, Savola S et al. A molecular diagnostic approach able to detect the recurrent genetic prognostic factors typical of presenting myeloma. Genes Chromosomes Cancer 2015; 54: 91-98.

$14 \mathrm{R}$ Development Core Team. $R$ : a language and environment for statistical computing. R Foundation for Statistical Computing: Vienna, Austria, 2010.

15 Walker BA, Leone PE, Chiecchio L, Dickens NJ, Jenner MW, Boyd KD et al. A compendium of myeloma-associated chromosomal copy number abnormalities and their prognostic value. Blood 2010; 116: e56-e65.

16 Van Wier S, Braggio E, Baker A, Ahmann G, Levy J, Carpten JD et al. Hypodiploid multiple myeloma is characterized by more aggressive molecular markers than non-hyperdiploid multiple myeloma. Haematologica 2013; 98: 1586-1592.

17 Bergsagel PL, Kuehl WM, Zhan F, Sawyer J, Barlogie B, Shaughnessy J Jr. Cyclin D dysregulation: an early and unifying pathogenic event in multiple myeloma. Blood 2005; 106: 296-303.

18 Palumbo A, Avet-Loiseau H, Oliva S, Lokhorst HM, Goldschmidt H, Rosinol L et al. Revised international staging system for multiple myeloma: a report from International Myeloma Working Group. J Clin Oncol 2015; 33: 2863-2869.

19 Smith LK, He Y, Park JS, Bieri G, Snethlage CE, Lin K et al. beta2-microglobulin is a systemic pro-aging factor that impairs cognitive function and neurogenesis. Nat Med 2015; 21: 932-937.

20 Greipp PR, San Miguel J, Durie BG, Crowley JJ, Barlogie B, Blade J et al. International staging system for multiple myeloma. J Clin Oncol 2005; 23: 3412-3420.

21 Sonneveld P, Avet-Loiseau H, Lonial S, Usmani S, Siegel D, Anderson KC et al. Treatment of Multiple Myeloma with high-risk cytogenetics: a consensus of the International Myeloma Working Group. Blood 2016; 127: 2955-2962.

22 Kyle RA, Rajkumar SV. An overview of the progress in the treatment of multiple myeloma. Expert Rev Hematol 2014; 7: 5-7.

23 Chu Y, Soberon V, Glockner L, Beyaert R, Massoumi R, van Loo G et al. A20 and CYLD do not share significant overlapping functions during $B$ cell development and activation. J Immunol 2012; 189: 4437-4443.

24 Annunziata CM, Davis RE, Demchenko Y, Bellamy W, Gabrea A, Zhan F et al. Frequent engagement of the classical and alternative NF-kappaB pathways by diverse genetic abnormalities in multiple myeloma. Cancer Cell 2007; 12: $115-130$.

25 Keats JJ, Fonseca R, Chesi M, Schop R, Baker A, Chng WJ et al. Promiscuous mutations activate the noncanonical NF-kappaB pathway in multiple myeloma. Cancer Cell 2007; 12: 131-144.

26 Tornatore L, Sandomenico A, Raimondo D, Low C, Rocci A, Tralau-Stewart C et al. Cancer-selective targeting of the NF-kappaB survival pathway with GADD45beta/ MKK7 inhibitors. Cancer Cell 2014; 26: 495-508.

27 Lalaoui N, Hanggi K, Brumatti G, Chau D, Nguyen NY, Vasilikos L et al. Targeting p38 or MK2 enhances the anti-leukemic activity of smac-mimetics. Cancer Cell 2016; 29: 145-158.

28 Demchenko YN, Brents LA, Li Z, Bergsagel LP, McGee LR, Kuehl MW. Novel inhibitors are cytotoxic for myeloma cells with NFkB inducing kinase-dependent activation of NFkB. Oncotarget 2014; 5: 4554-4566.

29 Hayden MS, Ghosh S. NF-kappaB, the first quarter-century: remarkable progress and outstanding questions. Genes Dev 2012; 26: 203-234.

30 Keats JJ, Reiman T, Maxwell CA, Taylor BJ, Larratt LM, Mant MJ et al. In multiple myeloma, $\mathrm{t}(4 ; 14)(\mathrm{p} 16 ; \mathrm{q} 32)$ is an adverse prognostic factor irrespective of FGFR3 expression. Blood 2003; 101: 1520-1529.

31 Smadja NV, Bastard C, Brigaudeau C, Leroux D, Fruchart CGroupe Francais de Cytogenetique $\mathrm{H}$. Hypodiploidy is a major prognostic factor in multiple myeloma. Blood 2001; 98: 2229-2238.

32 Boyd KD, Ross FM, Tapper WJ, Chiecchio L, Dagrada G, Konn ZJ et al. The clinical impact and molecular biology of del(17p) in multiple myeloma treated with 
conventional or thalidomide-based therapy. Genes Chromosomes Cancer 2011; 50: 765-774

33 Sawyer JR, Tian E, Heuck CJ, Epstein J, Johann DJ, Swanson CM et al. Jumping translocations of $1 \mathrm{q} 12$ in multiple myeloma: a novel mechanism for deletion of $17 \mathrm{p}$ in cytogenetically defined high-risk disease. Blood 2014; 123: 2504-2512.

34 Chagtai T, Zill C, Dainese L, Wegert J, Savola S, Popov S et al. Gain of 1q as a Prognostic Biomarker in Wilms Tumors (WTs) treated with preoperative chemotherapy in the International Society of Paediatric Oncology (SIOP) WT 2001 trial: a SIOP Renal Tumours Biology Consortium Study. J Clin Oncol 2016; 34: 3195-3203.

35 Fonseca R, Van Wier SA, Chng WJ, Ketterling R, Lacy MQ, Dispenzieri A et al. Prognostic value of chromosome 1q21 gain by fluorescent in situ hybridization and increase CKS1B expression in myeloma. Leukemia 2006; 20: 2034-2040.

36 Touzeau C, Dousset C, Le Gouill S, Sampath D, Leverson JD, Souers AJ et al. The $\mathrm{BCl}-2$ specific $\mathrm{BH} 3$ mimetic ABT-199: a promising targeted therapy for $\mathrm{t}(11 ; 14)$ multiple myeloma. Leukemia 2014; 28: 210-212.

37 Baker GL, Landis MW, Hinds PW. Multiple functions of D-type cyclins can antagonize pRb-mediated suppression of proliferation. Cell Cycle 2005; 4: 330-338.

38 Bergsagel PL, Kuehl WM. Molecular pathogenesis and a consequent classification of multiple myeloma. J Clin Oncol 2005; 23: 6333-6338.
39 Dimopoulos MA, Oriol A, Nahi H, San-Miguel J, Bahlis NJ, Usmani SZ et al. Daratumumab, lenalidomide, and dexamethasone for multiple myeloma. $N$ Engl J Med 2016; 375: 1319-1331.

40 Rajkumar SV, Harousseau JL. Next-generation multiple myeloma treatment: a pharmacoeconomic perspective. Blood 2016; 128: 2757-2764.

41 Moreau P, Masszi T, Grzasko N, Bahlis NJ, Hansson M, Pour L et al. Oral ixazomib, lenalidomide, and dexamethasone for multiple myeloma. N Engl J Med 2016; 374: 1621-1634.

42 Stewart AK, Rajkumar SV, Dimopoulos MA, Masszi T, Spicka I, Oriol A et al. Carfilzomib, lenalidomide, and dexamethasone for relapsed multiple myeloma. N Engl J Med 2015; 372: 142-152.

(c) (1)

This work is licensed under a Creative Commons Attribution 4.0 International License. The images or other third party material in this article are included in the article's Creative Commons license, unless indicated otherwise in the credit line; if the material is not included under the Creative Commons license, users will need to obtain permission from the license holder to reproduce the material. To view a copy of this license, visit http://creativecommons.org/licenses/ by/4.0/

(c) The Author(s) 2018

Supplementary Information accompanies this paper on the Leukemia website (http://www.nature.com/leu) 